\title{
Universal Geometric Condition for the Transverse Instability of Solitary Waves
}

\author{
Thomas J. Bridges \\ Department of Mathematics and Statistics, University of Surrey, Guildford, Surrey GU2 5XH, United Kingdom
}

(Received 20 August 1999)

\begin{abstract}
Transverse instabilities correspond to a class of perturbations traveling in a direction transverse to the direction of the basic solitary wave. Solitary waves traveling in one space direction generally come in one-parameter families. We embed them in a two-parameter family and deduce a new geometric condition for transverse instability of solitary waves. This condition is universal in the sense that it does not require explicit properties of the solitary wave-or the governing equation. In this paper the basic idea is presented and applied to the Zakharov-Kuznetsov equation for illustration. An indication of how the theory applies to a large class of equations in physics and oceanography is also discussed.
\end{abstract}

PACS numbers: 05.45.Yv, 47.35. $+\mathrm{i}, 47.54 .+\mathrm{r}$

The fundamental model and the first-discovered example of the transverse instability of solitary waves is the instability of the Korteweg-de Vries (KdV) solitary wave in the Kadomtsev-Petviashvili (KP) equation,

$$
\begin{aligned}
u_{t}+u u_{x}+u_{x x x} & =v_{y} \\
\text { and } \quad v_{x}+\sigma u_{y} & =0, \quad \sigma= \pm 1,
\end{aligned}
$$

when $\sigma=-1$. This equation and a perturbation theory for transverse instability was proposed by Kadomtsev and Petviashvili in [1]. Historically this is the first paper to consider the transverse instability of solitary waves. Shortly thereafter it was recognized by Zakharov [2] that the KP equation is a completely integrable Hamiltonian partial differential equation, and therefore the transverse instability result for KP and other integrable models could be deduced a number of different ways, including explicit calculation. This led to a theory for transverse instability of a wide range of integrable systems (see Kuznetsov et al. [3] for a review). In general the theories since then can be classified into three categories: (a) theories that use integrability [3], (b) theories based on modulation equations [4-7], and (c) approximate theories based on Rayleigh-Ritz approximations or direct numerics $[6,8,9]$.

In this Letter we present a new geometric condition for the transverse instability of solitary waves of Hamiltonian evolution equations which is exact, does not rely on an explicit form for the wave, and does not rely on the particular form of the Hamiltonian evolution equation nor on integrability. To illustrate the basic idea, consider the Zakharov-Kuznetsov (ZK) equation [10] in two space dimensions

$$
u_{t}+u u_{x}+u_{x x x}+u_{y y x}=0 .
$$

The basic $y$-independent $\mathrm{KdV}$ solitary wave state is given by

$$
\begin{aligned}
u(x, y, t)=\hat{u}(\theta ; c) & =3 c \operatorname{sech}^{2}\left(\frac{1}{2} \sqrt{c} \theta\right), \\
\theta & =x-c t .
\end{aligned}
$$

Let $u(x, y, t)=\hat{u}(\theta ; c)+\operatorname{Re}\left[v(\theta ; \lambda, \beta) e^{\lambda t+i \beta y}\right]$ and linearize (1) about the one-parameter family of states $\hat{u}$ :

$$
v_{\theta \theta \theta}+(\hat{u}-c) v_{\theta}+\left(\lambda-\beta^{2}+\hat{u}_{\theta}\right) v=0 .
$$

For fixed $\hat{u}$ and $c$, the state $\hat{u}$ is said to be transverse unstable if the spectral problem (3) has a square-integrable solution with $\operatorname{Re}(\lambda)>0$ and $\beta \in \mathbb{R}$ (and $\beta \neq 0$ ). For the $\mathrm{ZK}$ equation (1), transverse instability for small $\beta$ was first shown in [11], and then for a wide range of $\beta$ in $[8,12,13]$. Here we will use this transverse instability to illustrate the new geometric condition and then discuss the general nature of this condition and its application to other Hamiltonian partial differential equations (PDEs).

The one-parameter family of solitary waves (2) can be parametrized by $c$ or by the value of the momentum $A(\hat{u})=\int_{-\infty}^{+\infty} \hat{u}(\theta ; c)^{2} d \theta$. Deform the state (2) by letting $\theta=x+\ell y-c t$; then $\hat{u}$ is deformed to

$$
\begin{aligned}
\hat{u}(\theta ; c, \ell) & =3 c \operatorname{sech}^{2}\left[\frac{1}{2}\left(\frac{c}{1+\ell^{2}}\right)^{1 / 2} \theta\right], \\
\theta & =x+\ell y-c t .
\end{aligned}
$$

This is a two-parameter family of solitary waves whose limit as $\ell \rightarrow 0$ recovers (2). It appears to be a trivial rotation that can be eliminated by a rotation of coordinates. However, it is the rate of change of this solution (and the rate of change of a pair of associated functions) that provide the information needed: i.e., rotate, take derivatives, rotate back.

Much the same way that the momentum $A(\hat{u})$ is associated with the parameter $c$, we will show that there is a second function $B(\hat{u})$ associated with the parameter $\ell$. The main result to be presented here is the following: There is a natural two-parameter $(c, \ell)$ embedding of unidirectional solitary waves with associated dual functions $A$ and $B$ and moreover, if

$$
\operatorname{det}\left[\begin{array}{ll}
\frac{\partial A}{\partial c} & \frac{\partial A}{\partial \ell} \\
\frac{\partial B}{\partial c} & \frac{\partial B}{\partial \ell}
\end{array}\right]>0,
$$

when $A$ and $B$ are evaluated on the two-parameter family of solitary waves, the basic state is transverse unstable. Moreover, this result applies in general to solitary wave states of -integrable or nonintegrable-Hamiltonian evolution equations. 
The key to deducing the form of the function $B$ and the condition (5) is to reformulate Hamiltonian evolution equations as Hamiltonian systems on a multisymplectic structure. Such structures have been used to generalize symplectic structure [14], to study the bifurcation and stability of periodic and quasiperiodic patterns $[15,16]$, and to study the longitudinal instability of solitary waves and fronts [17]. Here we show that the transverse symplectic structure encodes information about the transverse instability of solitary waves.

A Hamiltonian system on a multisymplectic structurein two space dimensions and time-takes the canonical form

$$
\mathbf{M} Z_{t}+\mathbf{K} Z_{x}+\mathbf{L} Z_{y}=\nabla S(Z), \quad Z \in \mathbb{R}^{n},
$$

with $\mathbf{M}, \mathbf{K}$, and $\mathbf{L}$ constant $n \times n$ skew-symmetric matrices and $\nabla S$ is the gradient of $S$ with respect to the standard inner product on $\mathbb{R}^{n}$, which will be denoted by $\langle\cdot, \cdot\rangle$. The formulation (6) separates the equation in such a way that

$$
\begin{aligned}
\mathbf{M} & =\left[\begin{array}{rrrrrr}
0 & 0 & 0 & 0 & 0 & 0 \\
0 & 0 & 0 & -1 & 0 & 0 \\
0 & 0 & 0 & 0 & 0 & 0 \\
0 & 1 & 0 & 0 & 0 & 0 \\
0 & 0 & 0 & 0 & 0 & 0 \\
0 & 0 & 0 & 0 & 0 & 0
\end{array}\right], \\
\mathbf{L} & =\left[\begin{array}{rrrrrr}
0 & 0 & 0 & 0 & 0 & 0 \\
0 & 0 & 0 & 0 & 0 & -1 \\
0 & 0 & 0 & 0 & 1 & 0 \\
0 & 0 & 0 & 0 & 0 & 0 \\
0 & 0 & -1 & 0 & 0 & 0 \\
0 & 1 & 0 & 0 & 0 & 0
\end{array}\right]
\end{aligned}
$$

there are distinct symplectic operators associated with time (M), the $x$ direction $(\mathbf{K})$, and the transverse direction $y(\mathbf{L})$. To illustrate this formulation, we will multisymplectify the generalized ZK equation; generalize (1) to

$$
2 u_{t}+f(u)_{x}+u_{x x x}+u_{y y x}=0,
$$

where $f(u)=F^{\prime}(u)$ and $F(u)$ is some given smooth function (and the 2 is added for notational convenience). Introduce new variables

$$
Z=(p, u, q, \phi, v, w) \in \mathbb{R}^{6},
$$

with definitions

$$
u=\phi_{x}, \quad v=u_{x}-q_{y} \quad \text { and } \quad w=u_{y}+q_{x} .
$$

Then the ZK is recovered with $w_{x}-v_{y}=0, u_{t}+p_{x}=$ 0 , and

$$
\phi_{t}+v_{x}+w_{y}=p-f(u) .
$$

However, combining these six equations together, they can be written in the form (7) with

$$
\begin{aligned}
\mathbf{K}=\left[\begin{array}{rrrrrr}
0 & 0 & 0 & -1 & 0 & 0 \\
0 & 0 & 0 & 0 & -1 & 0 \\
0 & 0 & 0 & 0 & 0 & -1 \\
1 & 0 & 0 & 0 & 0 & 0 \\
0 & 1 & 0 & 0 & 0 & 0 \\
0 & 0 & 1 & 0 & 0 & 0
\end{array}\right], \\
\text { and } \nabla S(Z)=\left[\begin{array}{c}
-u \\
f(u)-p \\
0 \\
0 \\
v \\
w
\end{array}\right] ;
\end{aligned}
$$

hence $S(Z)=\frac{1}{2}\left(v^{2}+w^{2}\right)-u p+F(u)$. Almost all Hamiltonian PDEs known to the author can be reformulated as Hamiltonian systems on a multisymplectic structure: the water wave problem [14,15], geophysical flows [15], the KP equation [18], fluid-structure interaction [15], pattern formation PDEs [16], wave equations [17], and Boussinesq models [17], for example. Such a multisymplectification amounts to a decomposition of the usual Hamiltonian function into (space) directional components.

The geometric condition for transverse instability will be deduced from the canonical form (6). Let $\hat{Z}(\theta ; c, \ell)$ with $\theta=x+\ell y-c t$ be any solitary wave solution of (6) which decays exponentially as $\theta \rightarrow \pm \infty$. Substitution into (6) shows that $\hat{Z}$ satisfies

$$
[\mathbf{K}+\ell \mathbf{L}-c \mathbf{M}] \hat{Z}_{\theta}=\nabla S(\hat{Z}) .
$$

Since we will need these functions in the sequel, we note that

$$
\mathcal{L} \hat{Z}_{\theta}=0, \quad \mathcal{L} \hat{Z}_{c}=-\mathbf{M} \hat{Z}_{\theta} \quad \text { and } \quad \mathcal{L} \hat{Z}_{\ell}=\mathbf{L} \hat{Z}_{\theta},
$$

obtained by differentiating (8), where

$$
\mathcal{L}=D^{2} S(\hat{Z})-[\mathbf{K}+\ell \mathbf{L}-c \mathbf{M}] \frac{d}{d \theta} .
$$

Another interesting feature of (8) is that it can be interpreted as the necessary condition for a constrained variational principle. Let

$$
\begin{aligned}
& A(\hat{Z})=-\frac{1}{2} \int_{-\infty}^{+\infty}\left\langle\mathbf{M} \hat{Z}_{\theta}, \hat{Z}\right\rangle d \theta, \\
& B(\hat{Z})=\frac{1}{2} \int_{-\infty}^{+\infty}\left\langle\mathbf{L} \hat{Z}_{\theta}, \hat{Z}\right\rangle d \theta,
\end{aligned}
$$

and $H(\hat{Z})=\int_{-\infty}^{+\infty}\left[S(\hat{Z})-\frac{1}{2}\left\langle\mathbf{K} \hat{Z}_{\theta}, \hat{Z}\right\rangle\right] d \theta$. Then $\hat{Z}$ can be characterized as a critical point of $H(\hat{Z})$ restricted to level sets of $A$ and $B$ with $c$ and $\ell$ as Lagrange multipliers. It follows from standard Lagrange multiplier theory that this state is nondegenerate precisely when

$$
\operatorname{det}\left[\begin{array}{cc}
A_{c} & A_{\ell} \\
B_{c} & B_{\ell}
\end{array}\right] \neq 0 .
$$

It remains to show the remarkable result that positivity of this determinant implies that the basic solitary wave is a 
transverse unstable solution of the evolution equation. To show this we need to formulate the linear stability problem.

Let $Z(x, y, t)=\hat{Z}(\theta ; c, \ell)+\operatorname{Re}\left[U(\theta ; \lambda, \beta) e^{\lambda t+i \beta y}\right]$, and substitute into (6) and linearize:

$$
\mathcal{L} U=\lambda \mathbf{M} U+i \beta \mathbf{L} U, \quad U \in \mathbb{C}^{n},
$$

where $\mathcal{L}$ is defined in (10). Now, $\mathcal{L}$ is a symmetric operator with $\hat{Z}_{\theta}$ in the kernel [see Eqs. (9) and (10)]. We assume that $\hat{Z}_{\theta}$ is the only square integrable element in the kernel of $\mathcal{L}$, which is generically the case. Then the solvability condition for (13) is

$$
\Delta(\lambda, \beta)=\llbracket \hat{Z}_{\theta}, \lambda \mathbf{M} U+i \beta \mathbf{L} U \rrbracket=0,
$$

where $\llbracket \cdot, \cdot \rrbracket=\int_{-\infty}^{+\infty}\langle\cdot, \cdot\rangle d \theta$, and $U$ is the solution of (13).

Expand (14) in a Taylor series in $\lambda$ and $\beta$ and note that

$$
\begin{array}{r}
U(\theta ; \lambda, \beta)=\mathbb{C}_{1}\left[\hat{Z}_{\theta}-\lambda \hat{Z}_{c}+i \beta \hat{Z}_{\ell}\right. \\
\left.+\mathcal{O}\left(|\lambda|^{2}+|\beta|^{2}\right)\right],
\end{array}
$$

where $\mathbb{C}_{1}$ is an arbitrary nonzero complex constant. The expression (15) can be verified by substituting it into (13) and using (9). Substituting (15) into (14) then leads to

$$
\begin{aligned}
\Delta(\lambda, \beta)= & \mathbb{C}_{1} \llbracket \hat{Z}_{\theta}, \lambda \mathbf{M}\left(\hat{Z}_{\theta}-\lambda \hat{Z}_{c}+i \beta \hat{Z}_{\ell}\right) \rrbracket \\
& +\mathbb{C}_{1} \llbracket \hat{Z}_{\theta}, i \beta \mathbf{L}\left(\hat{Z}_{\theta}-\lambda \hat{Z}_{c}+i \beta \hat{Z}_{\ell}\right) \rrbracket+\ldots
\end{aligned}
$$

or

$$
\begin{aligned}
\Delta(\lambda, \beta)= & \mathbb{C}_{1}\left[-A_{c} \lambda^{2}+\left(A_{\ell}+B_{c}\right) i \lambda \beta+B_{\ell} \beta^{2}\right] \\
& +o\left(|\lambda|^{2}+|\beta|^{2}\right), \quad \text { as }|\lambda|+|\beta| \rightarrow 0,
\end{aligned}
$$

where we have used the expressions

$$
\left(\begin{array}{cc}
+\llbracket \hat{Z}_{\theta}, \mathbf{M} \hat{Z}_{c} \rrbracket & +\llbracket \hat{Z}_{\theta}, \mathbf{M} \hat{Z}_{\ell} \rrbracket \\
-\llbracket \hat{Z}_{\theta}, \mathbf{L} \hat{Z}_{c} \rrbracket & -\llbracket \hat{Z}_{\theta}, \mathbf{L} \hat{Z}_{\ell} \rrbracket
\end{array}\right)=\left(\begin{array}{cc}
A_{c} & A_{\ell} \\
B_{c} & B_{\ell}
\end{array}\right),
$$

obtained by differentiating $A$ and $B$ in (11) and using the exponential decay of $\hat{Z}$ as $\theta \rightarrow \pm \infty$.

Solving (16) for $\lambda$ results in

$$
\lambda=i \frac{A_{\ell}}{A_{c}} \beta \pm \frac{\beta}{A_{c}} \sqrt{A_{c} B_{\ell}-A_{\ell} B_{c}}+\mathcal{O}\left(\beta^{2}\right) .
$$

Therefore if the condition (5) is met, there is a solution of the spectral problem (13) with $\operatorname{Re}(\lambda)>0$ and $|\beta| \neq 0$ with growth rate

$$
\operatorname{Re}(\lambda)=\operatorname{det}\left[\begin{array}{ll}
A_{c} & A_{\ell} \\
B_{c} & B_{\ell}
\end{array}\right] \frac{|\beta|}{\left|A_{c}\right|}+\mathcal{O}\left(|\beta|^{2}\right) .
$$

The precise form of the unstable eigenfunction is of the form $\operatorname{Re}\left[U(\theta ; \lambda, \beta) e^{\lambda t+i \beta y}\right]$ with $U(\theta ; \lambda, \beta)$ given to leading order in (15) with exponent (17). With the condition $|\beta| \ll 1$, the eigenfunction is a transverse long-wave perturbation.
For example, we apply this result to the generalized ZK equation in (7). The functions $A$ and $B$ are

$$
\begin{aligned}
& A(\hat{Z})=-\frac{1}{2} \int_{-\infty}^{+\infty}\left\langle\mathbf{M} \hat{Z}_{\theta}, \hat{Z}\right\rangle d \theta=\int_{-\infty}^{+\infty} \hat{u}^{2} d \theta, \\
& B(\hat{Z})=\frac{1}{2} \int_{-\infty}^{+\infty}\left\langle\mathbf{L} \hat{Z}_{\theta}, \hat{Z}\right\rangle d \theta=\ell \int_{-\infty}^{+\infty}\left(\hat{u}_{\theta}\right)^{2} d \theta .
\end{aligned}
$$

With $f(u)=u^{m+1} /(m+1)$ a solitary wave state is given by

$$
\begin{aligned}
\hat{u}(\theta ; c, \ell)= & {[c(m+1)(m+2)]^{1 / m} } \\
& \times \operatorname{sech}^{2 / m}\left[\frac{m}{\sqrt{2}}\left(\frac{c}{1+\ell^{2}}\right)^{1 / 2} \theta\right],
\end{aligned}
$$

and a straightforward calculation shows that

$$
\operatorname{det}\left[\begin{array}{ll}
A_{c} & A_{\ell} \\
B_{c} & B_{\ell}
\end{array}\right]=\gamma^{2}\left(\frac{4-m}{4+m}-\ell^{2}\right),
$$

where $\gamma^{2}$ is a strictly positive function of $(c, \ell)$. The expression (19) recovers the result that ZK waves $(m=1)$ with $\ell=0$ are unstable [11], that ZK waves $(m=1)$ with $\ell \neq 0$ are unstable for $\ell<\left(\frac{3}{5}\right)^{1 / 2}[12,13]$, and it shows that this angle decreases with increasing nonlinearity: unstable for $\ell<\left(\frac{4-m}{4+m}\right)^{1 / 2}, m=2,3$. As noted above, the instabilities predicted by the condition (5) are transverse long-wave perturbations. Therefore when the determinant in (5) is negative, the wave is stable to long-wave perturbations but may be unstable to transverse short-wave instabilities (i.e., $\beta$ not small). Indeed, numerical results of [13] show that when the transverse long-wave instability for ZK stabilizes, the wave is still unstable to a transverse short-wave instability.

In summary, given any solitary-wave solution which decays exponentially as $\theta \rightarrow \pm \infty$ of a Hamiltonian evolution equation which can be formulated as in (6), the condition (5) gives a sufficient condition for transverse instability: the condition for transverse instability is encoded in the two-parameter embedding of the solitary wave. This condition is universal in the sense that it uses only the existence and exponential decay of the solitary wave and does not depend on particular properties of the evolutionary PDE, only its Hamiltonian structure.

Two immediate generalizations are of interest. First, it is straightforward to generalize this theory to the case where there is more than one transverse direction. Second, when the PDE is reflection invariant in the transverse direction, for example, if $u(x,-y, t)$ is a solution whenever $u(x, y, t)$ is a solution of (1) or more generally if $\mathbf{R} Z(x,-y, t)$ is a solution of (6) whenever $Z(x, y, t)$ is a solution for some involution $\mathbf{R}$ (i.e., $\mathbf{R}^{2}=\mathbf{I}$ ), then it is straightforward to show that $A$ and $B$ satisfy $A(c,-\ell)=A(c, \ell)$ and $B(c,-\ell)=-B(c, \ell)$. A consequence of this symmetry is

$$
\lim _{\ell \rightarrow 0} \operatorname{det}\left[\begin{array}{cc}
A_{c} & A_{\ell} \\
B_{c} & B_{\ell}
\end{array}\right]=A_{c}(c, 0) \hat{B}(c, 0),
$$


where $\hat{B}(c, 0)=\lim _{\ell \rightarrow 0} \ell^{-1} B(c, \ell)$. In this case transverse instability for the $\ell=0$ case can be deduced from the one-parameter family of solitary waves. Indeed, the ZK equation is an example of such a system. There are many other examples with this property, particularly models for water waves: the KP equation-and indeed the full water wave equations.

Generalizations and applications to other PDEs are considered in [19]. In [14] it was shown that the water wave problem is multisymplectic and therefore the theory presented here extends to study transverse instability of the wide range of oceanographic solitary waves and details of this generalization are also considered in [19]. Here we will sketch another intriguing generalization which applies to nonlinear Schrödinger (NLS) models and its generalizations (coupled NLS, etc.). Consider the following basic NLS model:

$$
-i A_{t}+A_{x x}+\sigma A_{y y}+V^{\prime}\left(|A|^{2}\right)=0,
$$

where $V(\cdot)$ is some smooth function and $\sigma= \pm 1$. The known results on the transverse instability of the cubic NLS model rely on the fact that the spectral problem associated with the linearization can be analyzed exactly; see [20] and references therein. However, by introducing new coordinates it is straightforward to formulate this system as a multisymplectic system of the form (6) with $n=8$. Formulating the transverse stability problem leads to a generalization of the Vakhitov-Kolokolov condition [21,22] — which holds for longitudinal instabilities only - to a new condition similar to (5) which applies to transverse instabilities of NLS, coupled NLS systems, and other generalized NLS systems. The full details will be given elsewhere.
[1] B. B. Kadomtsev and V. I. Petviashvili, Sov. Phys. Dokl. 15, 539 (1970).

[2] V.E. Zakharov, JETP Lett. 22, 172 (1975).

[3] E. A. Kuznetsov, A. M. Rubenchik, and V.E. Zakharov, Phys. Rep. 142, 104 (1986).

[4] L. Ostrovsky and V. Shrira, Sov. Phys. JETP 44, 738 (1976).

[5] V. Shrira, Sov. Phys. JETP 52, 44 (1980).

[6] E. W. Laedke and K. H. Spatschek, Phys. Rev. Lett. 41, 1798 (1978).

[7] P. A. E. M. Janssen and J. J. Rasmussen, Phys. Fluids 26, 1279 (1983).

[8] M. A. Allen and G. Rowlands, J. Plasma Phys. 50, 413 (1993).

[9] H. Iwasaki, S. Toh, and T. Kawahara, Physica (Amsterdam) 43D, 293 (1990).

[10] V.E. Zakharov and E. A. Kuznetsov, Sov. Phys. JETP 39, 285-286 (1974).

[11] K. H. Spatschek, P. K. Shukla, and M. Y. Yu, Phys. Lett. 54A, 419 (1975).

[12] K. P. Das and F. Verheest, J. Plasma Phys. 41, 139 (1989).

[13] M. A. Allen and G. Rowlands, J. Plasma Phys. 53, 63 (1995).

[14] T. J. Bridges, Math. Proc. Camb. Philos. Soc. 121, 147 (1997).

[15] T. J. Bridges, Proc. R. Soc. London A 453, 1365 (1997).

[16] T. J. Bridges, Nonlinearity 11, 467 (1998).

[17] T. J. Bridges and G. Derks, Proc. R. Soc. London A 455, 2427 (1999).

[18] T. J. Bridges, Eur. J. Mech. B, Fluids 18, 493 (1999).

[19] T. J. Bridges (to be published).

[20] E. A. Kuznetsov and S. K. Turitsyn, Sov. Phys. JETP 67, 1583 (1989).

[21] N. G. Vakhitov and A. A. Kolokolov, Izv. Vuz. Radiofiz. 16, 1020 (1973) [Radiophys. Quantum Elec. 16, 783 (1975)].

[22] L. Bergé, Phys. Rep. 303, 259 (1998). 